\title{
The Vacuum Deposition of Gold
}

\section{DEVELOPMENTS IN COATING TECHNIQUES AND APPLICATIONS}

\author{
Both the physical phenomena occurring in the vacuum deposition \\ techniques now in use and the problems involved in their appli- \\ cation were discussed at an international conference in London.
}

The vacuum deposition processes of thermal evaporation and cathodic sputtering make it possible to produce thin films of metal upon the surface of many different kinds of substrate. The discovery of the latter process was made by Sir William Grove as long ago as 1852, when he observed metallic deposits on the walls of his glow discharge tube near to the cathode, and it was first employed some fifty years later by Thomas Edison in the sputtering of gold on to his wax phonograph masters in order to provide a conductive surface for subsequent electrodeposition. Gold is in fact the only metal that can be applied in this way at moderately low pressures in air, and it was therefore used for coating non-metallic objects long before the availability of inert gases made it possible to use the process for a much wider range of metals. The optical industry was probably the first to make use of gold sputtering as a production process, while in more recent years a substantial application has developed in the production of heat reflective coatings of bismuth oxide and gold sputtered on glass for windows for aircraft and for buildings in warm climates. Plant is in fact now in use for the gold coating of individual sheets of glass in sizes up to two metres square.

With the development of high capacity diffusion pumps and the consequent achievement of very high vacuum conditions on a production scale, coating by simple thermal evaporation from a hot metal source became feasible and this process has thus greatly out-stripped sputtering in its breadth of application, although the rapid growth of microelectronics has led to an increased interest in sputtering to provide greater deposition control and improved physical characteristics in the film.

Both these broad types of vacuum coating processes have received a great deal of attention during the past ten years or so, and an enormous literature has been built up. While there has perhaps been relatively little change in the fundamentals of the deposition processes during this period, a great deal of research has been devoted to their improvement and to the development of sophisticated and partly automated equipment. There are now in fact many variations on the two broad themes of vacuum coating, and each requires specialised knowledge

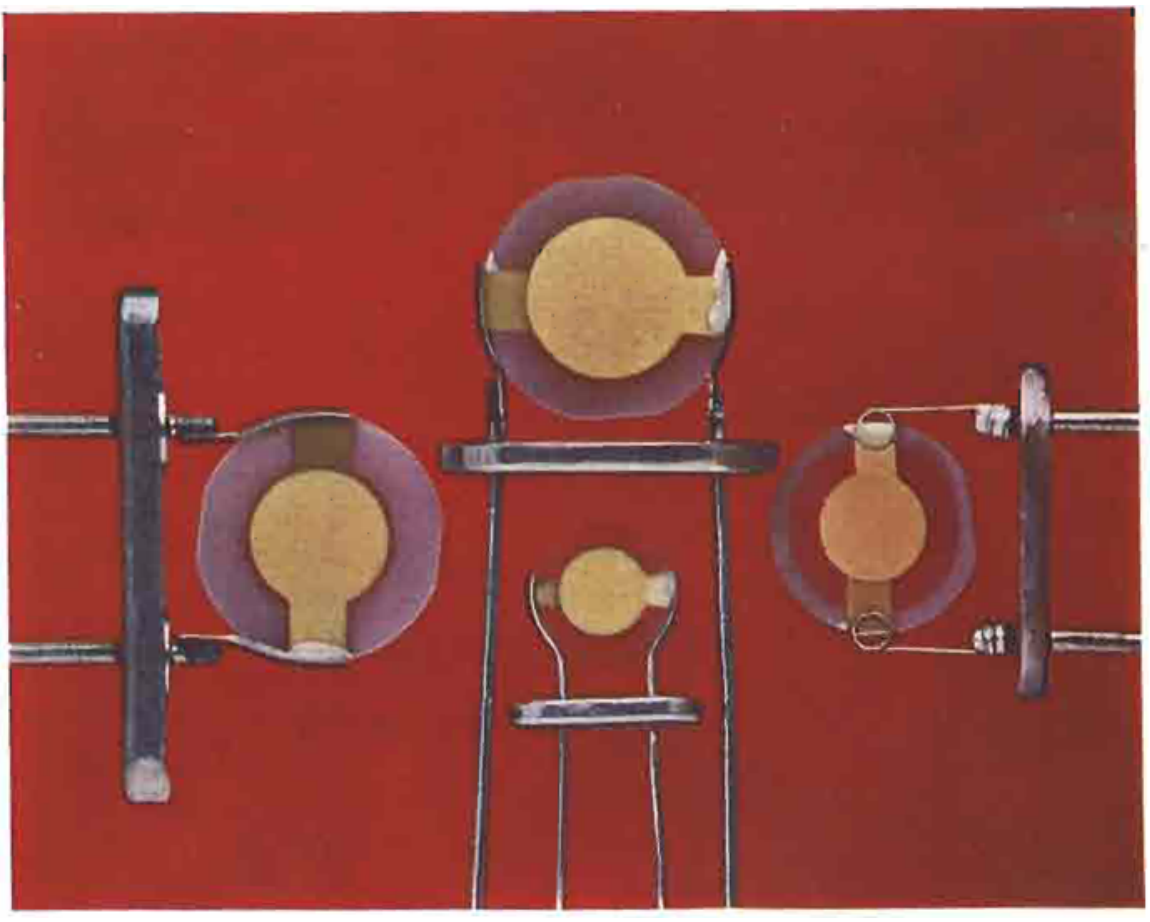

The piezo-electric effect causes quartz crystals to change their dimensions in the presence of an electric field and is the basis of quartz crystal oscillators designed to operate at fixed frequencies, which depend upon the crystal dimensions. These quartz oscillators, made by Cathodeon Crystals Limited, are used in small portable radio communicators such as those used by the police. Gold contact films to the crystal faces are sputtered on and further careful gold deposition by evaporation is used to calibrate the crystal oscillation to the desired frequency. 
In thị film integrated circuits the components such as resistors and capacitors are finally interconmected and provided with contact areas of gold, either by sputtering or by vacuum evaporation.

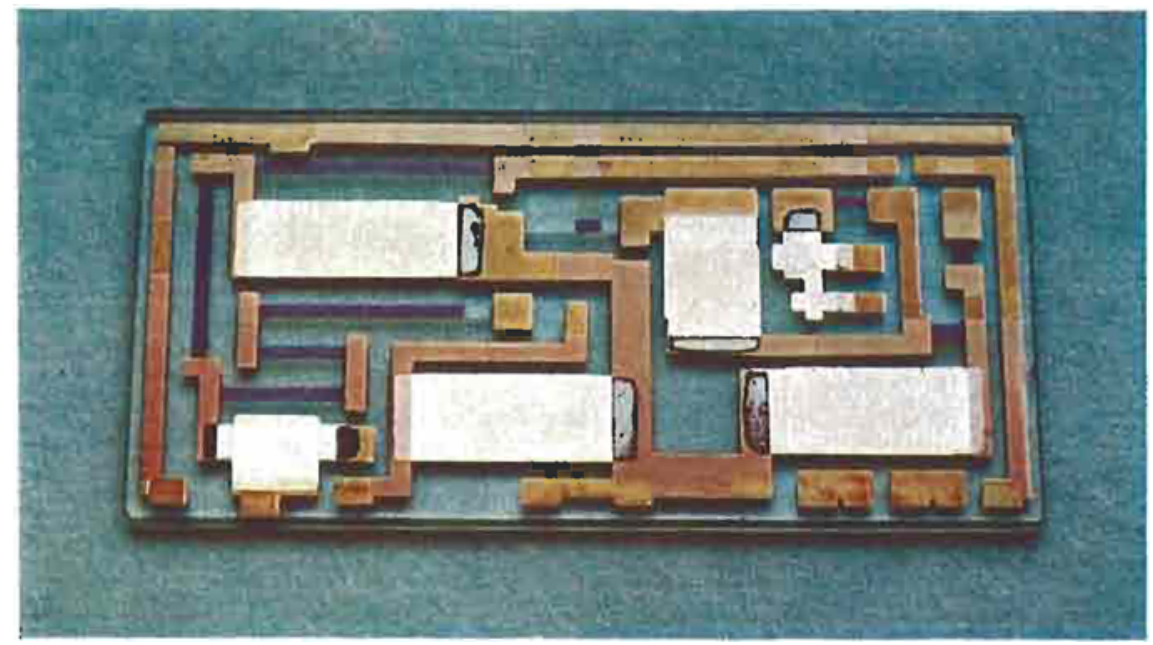

of the equipment available and its potentialities. In addition there has developed a better understanding of the basic physical and chemical mechanisms involved in these operations.

At a conference held in London in February, sponsored by Industrial Finishing and Surface Coatings, many aspects of the whole subject were discussed by over two hundred physicists and engineers. In opening the Conference, Dr $\mathrm{L}$. Holland, of Edwards High Vacuum, outlined the physical phenomena occurring in the vacuum deposition techniques currently in use and the technological problems that had to be solved to permit their industrial application. Although only two basic deposition processes were involved, there were many variations on their method of employment, and even some combinations of the processes.

In a survey of basic deposition techniques of vacuum evaporation, $\mathrm{Mr} \mathrm{B}$. J. Williams, also of Edwards High Vacuum, dealt with evaporation rates and vapour pressures, the construction of evaporation sources, the distribution of film thickness and the methods of measuring thickness. Mr T. I. Putner similarly reviewed the basic mechanisms of sputtering - the removal of material from a target by ion bombardment, its transport through a low density gas phase and deposition as a thin film on the substrate - and described some of its more recent applications. While thermally evaporated metal is ejected from the source with energy less than $1 \mathrm{eV}$, sputtered material energies are of the order of $10 \mathrm{eV}$, giving improved adhesion.

Applications of vacuum coating in the electrical and electronic industries were reviewed by $\mathrm{Mr} \mathrm{G}$. Siddall of the Electrical Research Association. The ability to deposit a thin film of metal in closely controlled amounts to a precise pattern with well defined electrical properties had been found extremely valuable to both component and circuit designers and the techniques of thermal evaporation and sputtering were being employed extensively.

A number of modern electronic devices - and some older ones - are based upon the piezo-electric effect whereby if certain materials such as quartz or a titanate ceramic be compressed, their opposite faces exhibit a potential difference. Conversely if a potential difference be applied across them, their dimensions change. A device of major importance based upon this effect is the quartz crystal used for the stabilisation of oscillatory circuits, the electrical frequency being stabilised by the frequency of vibration of the quartz. For such devices a thin conducting film which does not interfere seriously with the fundamental mode of vibration of the crystal is required, and this is often provided by sputtering a film of gold. The presence of this film does in fact change the frequency of vibration, and for accurate

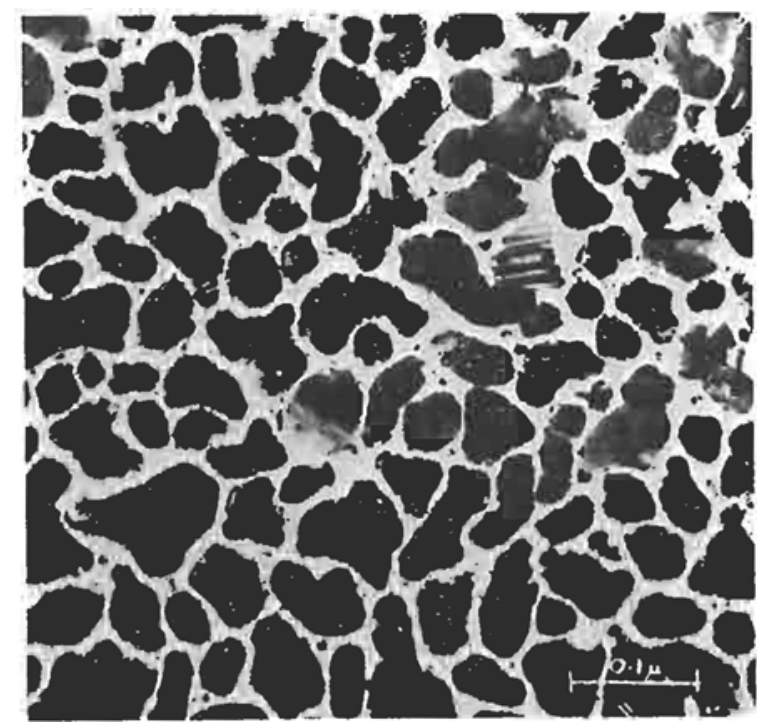

An electronmicrograph of the gold film forming the element of the strain gauge shown on page 32 . Its discontinuous structure makes it very sensitive to small changes brought about by mechanical strain. 


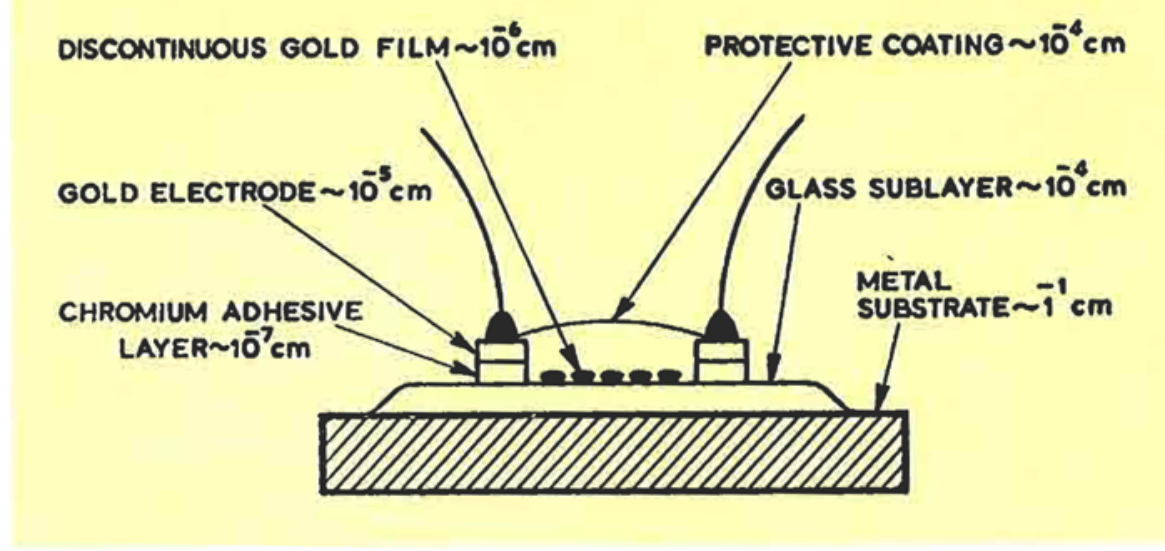

A diagrammatic view of the strain gauge developed by the Electrical Research Association. This consists of a thin film of gold with two gold planar electrodes deposited by vacuum evaporation and has the advantage of high sensitivity and of being able to be deposited directly on to the member under test.

work it is necessary to carry out a second deposition to calibrate the crystal. In modern equipment an evaporation technique is used, and both base coating and frequency calibration can be performed in the same apparatus and in the same vacuum cycle.

In thin film integrated circuits the inter-connections to the resistors and capacitors are produced by the evaporation of gold. This method gives stable contact surfaces readily capable of being soldered or welded. Similarly a number of semiconductor devices have their inter-connection layers or contacts provided by vacuum deposition of gold.

One further device based upon the vacuum evaporation of gold is a high sensitivity strain gauge developed by the Electrical Research Association. This consists of a thin film of gold with two thick planar electrodes, deposited on to a polished metal plate. The gold film forming the strain gauge element has a discontinuous or "island" structure as shown in the photomicrograph, the electrical resistance of the film lying mainly in the gaps between the particles. Conduction across these gaps is by quantum mechanical tunnelling and is very sensitive to small changes in gap width brought about by applied mechanical strains. This device is therefore highly sensitive, it eliminates the use of adhesives between the gauge and the structural member under test, and is small and of low cost.

\title{
Organo-Gold Hydrides?
}

\author{
D. J. Reynolds \\ Fulmer Researeh Institute, Stoke Poges, England.
}

Industrial usage of organometallic compounds as both catalytic and synthetic reagents has necessitated that the chemistry of a large number of compounds be examined. Many of these studies have led to the isolation and eventual characterisation of new and novel compounds. Among some of the most interesting of these, apart from those possibly capable of fixing molecular nitrogen and oxygen, are the transition metal hydride complexes. A number have been found capable of activating molecular hydrogen in homogeneous solutions, for example, $\mathrm{RhCl}\left(\mathrm{PPh}_{3}\right)_{3}$, operating by formation of a dihydro species with cis-hydrogens. Even apart from these in solution, a large number of solid hydride complexes have also been prepared. In the transition group of metals, apart from niobium, only the copper triad shows inability to form complex molecules or ions containing metal-hydrogen bonds.

One might question why gold should behave so differently in this respect from the other transition elements. The tremendous stability of the element itself naturally results in some loss in activity, and this is usually attributed to its overall percentage loss of $d$ character. Thus it may be better to classify the metal as a borderline transition metal, in line with its hydride

$\mathrm{Au}-\mathrm{H}$, although the actual existence of the latter is still uncertain. With an electronic configuration of $6 s^{25} d^{9}$ and a preference for the trivalent state in compound formation its most usual coordination number is four, with a square planar geometry. However, in certain circumstances higher coordination numbers are possible, i.e., five and six, with such stabilising ligands as diarsine for example.

Thus it may be pertinent to consider whether organo-gold (III) hydride complexes may be capable of existence possibly through ligand stabilisation of the metal atom. In normal gold alkyis, e.g., $R_{3} A u$, stabilisation is brought about by using nitrogen and phosphorus donors where the coordination stability achieved by bridging with halogen atoms, for example, is not possible. One would suspect a similar situation to hold for a compound such as $\mathrm{R}_{2} \mathrm{HAu}$; in this case donor stabilisation must also be possible. The real question then arises: are such compounds really non-existent? Considering the appreciable catalytic activity recently found for gold and its compounds in the areas of dehydrogenation and oxidation, little attention seems to have been given as yet to this other possible area of application. 